\title{
EXPERIMENTAL STUDY OF THE ION TRAPPING PHENOMENON IN TRISTAN-AR
}

\author{
S. Matsumoto, H. Fukuma, K. Ohmi, M. Tobiyama, E. Kikutani, M. Suetake, K. Satoh, Y. Funakoshi \\ High Energy Accelerator Research Organization (KEK), Oho 1-1, Tsukuba, Ibaraki, Japan
}

\section{Abstract}

The betatron oscillation of the electron beam in TRISTAN AR was observed in multi-bunch operation in which every a few tens of rf buckets are selected to be filled with beam. Transverse coupled bunch instability was observed and its growth time was measured by utilizing the feedback system. The betatron sideband spectrum were distorted when most of the selected buckets were filled. The conjecture of ion trapping explains the facts that we observed.

\section{INTRODUCTION}

During the high current operation study in TRISTANAR[1], we tried to store beam with full rf buckets (640 bunches) a few times. We found that we could store only $\sim 20 \mathrm{~mA}$ beam current or less, unless we use orbit feedback. At that moment, we observed the vertical beam oscillation whose frequency was $1-\delta \nu_{y}$, where $\delta \nu_{y}$ is the fractional part of the vertical tune $(\sim 0.23)$. Although this limitation was easily overcame by the feedback, however, we were interested in what gives it. This is the motivation of our study. We suspected this current limitation was coming from the coupled-bunch instability caused by the trapped ions. We identified the unstable modes and measured their growth time. We observed the betatron sideband spectrum to look for a signal of trapped ions on it. In this paper, we will report on these observations and discuss the results based on the conjecture of ion trapping.

The main machine parameters of AR, which are used in this paper, are listed below. The average vacuum pressure was a few $\times 10^{-7} \mathrm{~Pa}$.

\begin{tabular}{lcl} 
AR parameters & & \\
\hline Beam energy & $m_{e} c^{2} \gamma$ & $2.5 \mathrm{GeV}$ \\
RF frequency & $\omega_{\mathrm{rf}} / 2 \pi$ & $508.58 \mathrm{MHz}$ \\
Harmonic number & $h$ & 640 \\
Revolution frequency & $\omega_{\mathrm{rev}} / 2 \pi$ & $794.66 \mathrm{kHz}$ \\
Circumference & $C$ & $377.26 \mathrm{~m}$ \\
Horizontal emittance & $\epsilon_{x}$ & $43.6 \mathrm{~nm}$ \\
Horizontal tune & $\nu_{x}$ & 10.160 \\
Vertical tune & $\nu_{y}$ & 10.228
\end{tabular}

For simplicity, we use the averaged beam sizes which are defined as $\sigma_{x}=\sqrt{\epsilon_{x} \bar{\beta}_{x}}=510 \mu \mathrm{m}$, where $\bar{\beta}_{x}=C / 2 \pi \nu_{x}$ is the constant called the typical value of the horizontal betatron function. We assume $6 \% x y$ coupling and we get $\sigma_{y}=125 \mu \mathrm{m}$. In the followings of this paper, the beam sizes read these constant values.

\section{EXPERIMENTS AND RESULTS}

In our study, we operated the machine with 64 bunches (equally spaced, every 10 rf buckets) instead of with 640 bunches (to save time for the beam injection). According to the argument of the stability of the linearized motion of ions near the beam orbit, 64-bunch beam can trap all kinds of ions (singly ionized). Suppose the beam consists of $n_{b}$ identical bunches, which are equally separated from each other (by $C / n_{b}$ ), and each contains $N_{e}$ electrons. The bunch has the Gaussian distribution both in $x$ and $y$ whose r.m.s. are $\sigma_{x}$ and $\sigma_{y}$ respectively. The stability criterion of the ion (vertical) motion is

$$
\frac{4 \pi N_{e} r_{p} c}{\omega_{\mathrm{rev}} A} \frac{1}{\sigma_{y}\left(\sigma_{x}+\sigma_{y}\right)} \frac{1}{n_{b}} \leq 4,
$$

where $r_{p}$ is the classical proton radius. By using the parameters in the table, the formula is reduced to $I(\mathrm{~mA}) \leq$ $0.035 A n_{b}^{2}$ where $I$ is the total stored beam current. If $n_{b}=64$, all kinds of ions are trapped if $I \leq 140 \mathrm{~mA}$. Since the beam current was at most $I \sim 100 \mathrm{~mA}$ in our study, $n_{b}=64$ is enough.

We measured the growth time of the unstable mode by utilizing the transverse feedback system in AR[2]. By the trigger of the feedback off, the data taking was started to record the positions of every bunch turn to turn (for 1640 turns). The stored data was processed by FFT to see the spectrum and its time dependence. See Fig. 1. In this case, the initial beam current was $80 \mathrm{~mA}$. We can see the lower sideband at the frequency $2-\delta \nu_{y}$ grows up. The growth looks linear rather than exponential. When we start with $110 \mathrm{~mA}$, we found the sideband at $3-\delta \nu_{y}$ grew up linearly. The summary of our observations is

- $m=12$ mode is unstable at $80 \mathrm{~mA}$,

- $m=13$ mode is unstable at $110 \mathrm{~mA}$,

- linear growth.

One possible explanation of this coupled-bunch instability is the resistive wall instability. We estimated its growth time to be a few tens msec or less but this is too slow compared with our observation in Fig. 1. If resistive wall instability occurs, the maximum growth always appears in $m=11$ mode and this means the sideband at $1-\delta \nu_{y}$ has always dominant growth. However, this was not true in our observation. The impedance of the rf cavities may also be another source of the instability. However, the impedance calculation shows the rf cavities give very small growth even when we operate the machine with its maximum beam current $(\sim 500 \mathrm{~mA})$. 


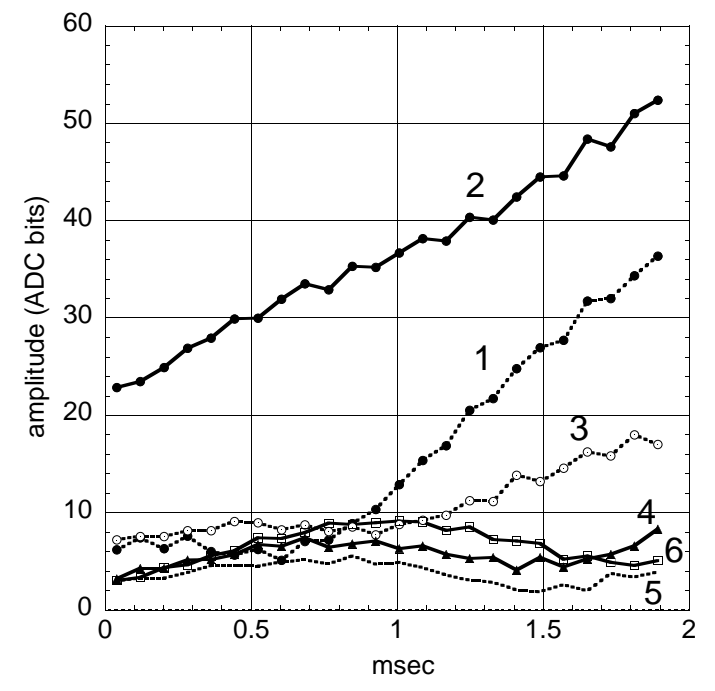

Figure 1: A result of the measurement of growth time. The growth of the betatron lower sidebands, $n-\delta \nu_{y}$ are plotted for the case $n=1,2, \ldots, 6$. We did not find a significant growth at the lower sidebands of $n \geq 4$ nor at all of the upper sidebands. Ordinate is the amplitude of the betatron sidebands and abscissa is elapsed time after the feedback being off. We see $n=1$ and $n=3$ sidebands also grow.

We now consider whether the observed mode instability can be induced by ion tapping or not. If the ions are produced and trapped by the stored beam, the transverse motion of the ions and the betatron motion of the beam are coupled. The 2-beam model[3] is the simplest model to describe this coupled oscillating system. In this model, both the beam and ions are uniformly distributed along the ring (they have constant line densities) and their transverse distributions are Gaussian of same dimensions both in $x$ and $y\left(\sigma_{x}\right.$ and $\left.\sigma_{y}\right)$. If the oscillation is small, the transverse oscillation of the ions is $\propto \exp (i \Omega t)$ while the beam oscillates $\propto \exp \{i(m \theta-\Omega t)\}$, where $m$ is the transverse mode index of the multiple bunches and $0<\theta<2 \pi$ is the azimuthal coordinate along the ring. The frequency $\Omega$ is the solution to the mode equation[3]

$$
\left(\Omega^{2}-\omega_{i}^{2}\right)\left\{\left(\Omega-m \omega_{\mathrm{rev}}\right)^{2}-\nu_{y}^{2} \omega_{\mathrm{rev}}^{2}-\omega_{e}^{2}\right\}=\omega_{e}^{2} \omega_{i}^{2} .
$$

The parameters in Eq. (2) are $\omega_{i}^{2}=\lambda_{e} r_{p} c^{2} / A \sigma_{y}\left(\sigma_{x}+\sigma_{y}\right)$ and $\omega_{e}^{2}=\lambda_{i} r_{e} c^{2} / \gamma \sigma_{y}\left(\sigma_{x}+\sigma_{y}\right)$, where $r_{e}$ is the classical electron radius and $\lambda_{e}$ and $\lambda_{i}$ are the line density of the electrons and of the ions along the ring respectively. The former, $\lambda_{e}$, reads $N_{e} n_{b} / C$ in our case. We consider only $\mathrm{CO}^{+}$since it is expected to be dominant among the ions in the ring.

Eq. (2) has four solutions and two of them can be complex, if $m \geq 11$. The complex solutions imply the system is unstable (2-beam instability). If this occurs, the oscillation grows exponentially. In Fig. 2, the frequency spectra of beam-ion system in the cases of $m=11,12$ and 13 are shown. It is notable that our observation of the unstable modes basically agrees with the model predictions on what mode is unstable and on where (how much beam current) it occurs. Note that the model predicts $m=11$ mode is unstable just above $10 \mathrm{~mA}$. This instability might cause the current limitation that we observed in 640-bunch operation. The oscillation at the sideband $1-\delta \nu_{y}$ will grow and this also meets our observation.

However, the growth rate of the unstable modes predicted by the model is the order of $\mu \mathrm{sec}$, which is extremely fast compared with our observations. Of course, the growth rate by the model is false, because we could stabilize the beam by the orbit feedback, which gives the damping rate about $1 \mathrm{msec}$. A weak-strong simulation (in which ions are treated by macroparticles while the beam is rigid) on the 2beam instability indicates that actual growth rate becomes smaller due to the ions decoherence by the nonlinear force by the beam[4]. Since ion decoherence is not included in the 2-beam model and this should be the one reason the model disagrees with our observation of the growth time.

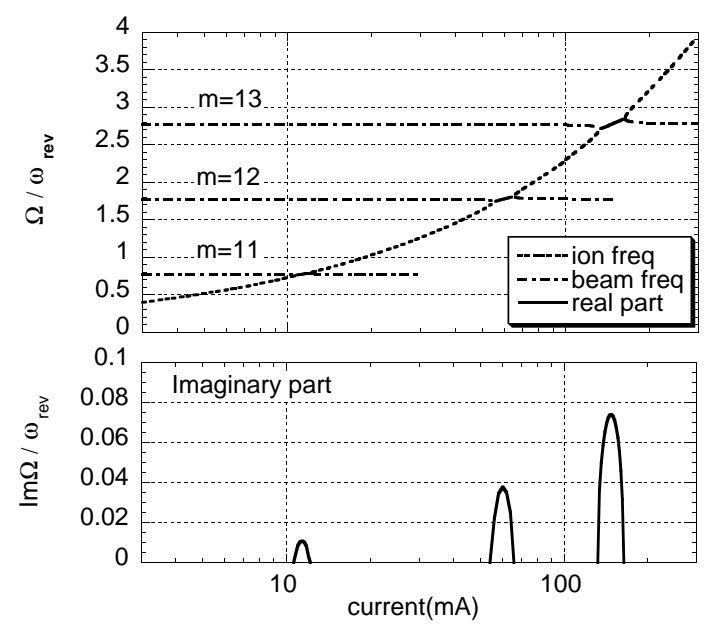

Figure 2: 2-beam instability by $\mathrm{CO}^{+}$ions. We assume $\lambda_{i} / \lambda_{e}=1 \times 10^{-3}$. If the solution is complex, both its real and imaginary part are shown. The mode $m=11$ is unstable in the beam current range from 10 to $12 \mathrm{~mA}$. Similarly, the model predicts $m=12$ and $m=13$ modes are unstable from 56 to $64 \mathrm{~mA}$ and from 134 to $162 \mathrm{~mA}$ respectively.

Next we observed the signal from a button pickup with a network analyzer to see the tune shift due to the ion trapping. We observed the spectrum of a sideband during the injection process. The beam was injected into the 64 buckets sequentially. In Fig. 3, we show three examples of spectrum at $4-\delta \nu_{y}$. Compare the spectrum at 20 buckets being filled (top picture) and that at 50 buckets (middle picture). The spectrum became fater and fatter (but the frequency of the peak was almost kept) and finally the spectrum was suddenly distorted like the bottom picture just before the injection was over (at around 60 buckets were filled). We see that the peak of the spectrum is at $2.9856 \mathrm{MHz}$ in the top picture while it is at $2.9843 \mathrm{MHz}$ in the bottom. The 


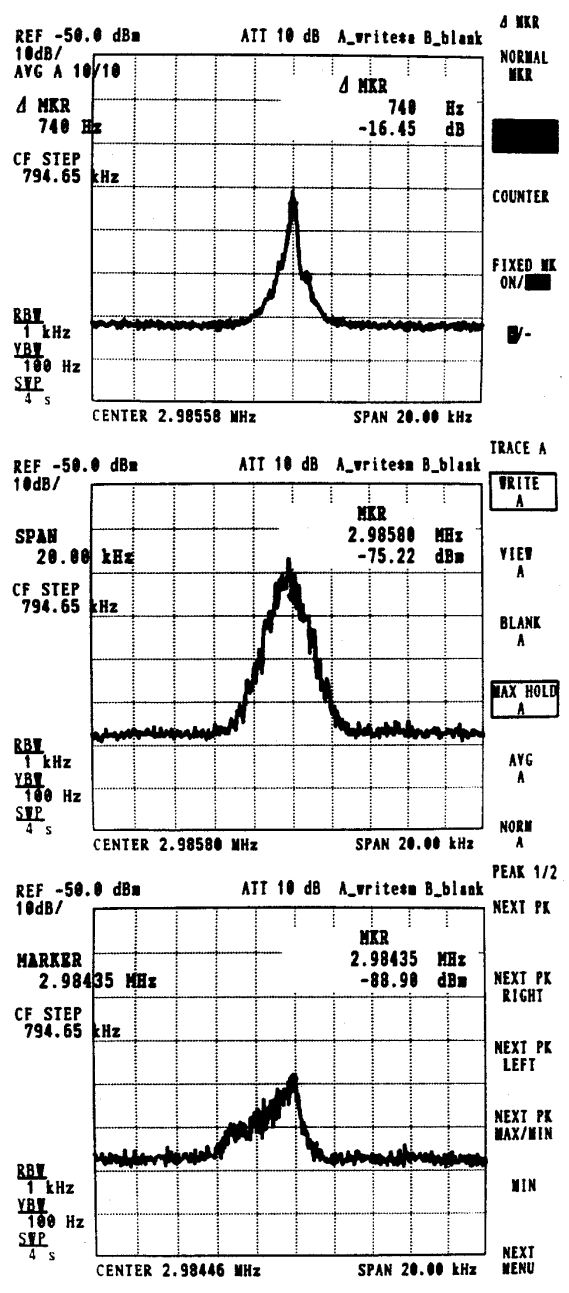

Figure 3: The pictures of the display of network analyzer to observe the spectrum of the betatron sideband at $4-$ $\nu_{y}$. Each picture shows the spectrum observed when 20 buckets(Top), 50 buckets(middle), or 64 buckets(bottom) were filled. The span of each picture is $20 \mathrm{kHz}$. The bunch current was $0.65 \mathrm{~mA}$ ( $42 \mathrm{~mA} / 64$ bunches).

peak was shifted by $1.3 \mathrm{kHz}$ by this distortion.

We performed same observations for 32-bunch, 16-bunch and 4-bunch operation mode, keeping the total beam current. In the 32-bunch mode (bunch current was $\sim 1.3 \mathrm{~mA}$ ), the spectrum was again changed as in the 64-bunch mode. It is implicative that this occurred when about 30 buckets were filled. In the other operation modes, we didn't see the change. This kind of distorted spectrum was observed in other machine[5]. It is natural to understand this spectrum change is induced by the additional focusing by the trapped ions. We tried to reproduce the "trapped signal", the spectrum like the bottom picture in Fig. 3, by the weakstrong simulation. The result is shown in Fig. 4. A spectrum which looks similar to the trapped signal has been obtained.
Finally, let us estimate $\lambda_{i}$ from tune shift by the formula

$$
\delta \nu_{y} \approx \frac{r_{e}}{\gamma} \frac{\bar{\beta}_{y} C}{4 \pi} \frac{1}{\sigma_{y}\left(\sigma_{x}+\sigma_{y}\right)} \lambda_{i},
$$

where $\bar{\beta}_{y}$ is the typical betatron function $(\sim 6 \mathrm{~m})$. By Eq. (3), we find the total number of ions in the ring is $\lambda_{i} C \sim 3.3 \times 10^{8}$ if the tune shift is $1.3 \mathrm{kHz}$. The number of beam electrons is $7.9 \times 10^{9} I(\mathrm{~mA})$. In Fig. $3, I=42 \mathrm{~mA}$ and we find the ratio $\lambda_{i} / \lambda_{e}$ is $1 \times 10^{-3}$.

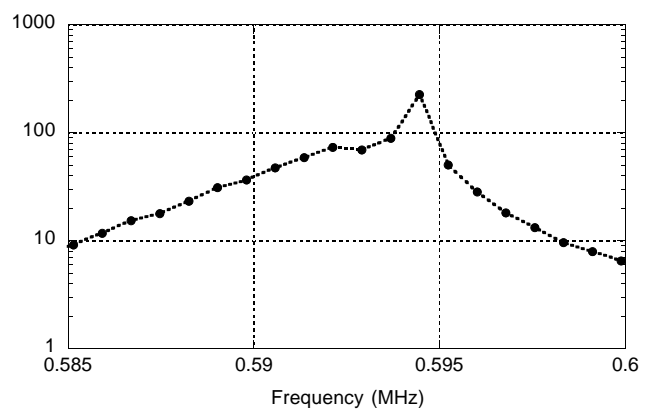

Figure 4: Weak-strong simulation result on the response (the amplitude of forced oscillation) of the beam. The beam is shaken at the frequency near $1-\delta \nu_{y}$ sideband. The beam consists of 64 bunches and the beam current is $15 \mathrm{~mA}$.

\section{CONCLUSION}

The facts we observed here suggest that the trapped ions do exist in the multi-bunch operation in AR. The 2-beam model is useful to understand the mechanism of the instability, however, the numerical simulation is necessary for clarifying the detailed mechanism, since the strong nonlinearity in the ion motion plays a role.

\section{ACKNOWLEDGEMENTS}

We are grateful to TRISTAN OPERATION GROUP for the support of this experiment.

\section{REFERENCES}

[1] Y. Funakoshi et al.: 'High beam current experiments for the KEKB conducted at TRISTAN', in these proceedings.

[2] M. Tobiyama and E. Kikutani, 'Study of Bunch by Bunch Feedback System in TRISTAN-AR', in these proceedings.

[3] E. Keil, B. Zotter, CERN-ISR-TH/71-58(1971); D. G. Koshkarev, P. R. Zenkevich, Particle Accelerators 3, 1 (1972) .

[4] K. Ohmi, KEK Preprint 96-160, to be appeared in Phys. Rev. E 55.

[5] D. Sagan and A. Temnykh, Nucl. Instr. Meth. A 344 (1994)459. 